\title{
Effects of pressure waves on the stability of flames propagating in tubes
}

\author{
Huahua Xiao*, Ryan W. Houim, Elaine S. Oran
}

Department of Aerospace Engineering, University of Maryland, College Park, Maryland, 20742, USA

${ }^{*}$ Corresponding author: Huahua Xiao

Mailing address: 2116 J.M. Patterson Building, College Park, MD 20742, USA

Emails: hhxiao@umd.edu, huahuahsiao@gmail.com

Colloquia: LAMINAR FLAMES; EXPLOSIONS

Total length and method: 5810 words, Method 1

\section{Word equivalent lengths:}

Main text: 3463 words

References: 489 words

Figure 1: 791 words

Figure 2: 188 words

Figure 3: 359 words

Figure 4: 368 words

Table 1: 152 words

Color reproduction: No, we do not want color reproduction of graphics and agree to reproduction in black and white in the printed version 


\section{Abstract}

Numerical simulations were performed to study the effects of pressure waves on the evolution and stability of flames propagating in tubes. The model is the fully compressible reactive Navier-Stokes equations coupled to a calibrated one-step chemical-diffusive model for combustion in a stoichiometric hydrogen-air mixture. The numerical solution method is high order in space and time, and adaptive mesh refinement provides adequate resolution of flames, boundary layers, acoustic waves, and all of their interactions. The influence of tube length scale and aspect ratio was examined for a range of tube sizes that produce tulip flames (TFs) and series of distorted tulip flames (DTFs). The simulations show that pressure waves and acoustic properties of the tube play an important role in the flame evolution, specifically in the formation and evolution of the series of increasingly wrinkled DTFs. A time scale and pressure wave analysis of the results, combined with a linear analysis to give the growth rate of the Rayleigh-Taylor instability (RTI), shows that the RTI is a primary cause for the initiation of DTFs. Keywords: Premixed flame; Distorted tulip flame; Flame-pressure wave interaction; Pressure amplification; Rayleigh-Taylor instability

\section{Introduction}

Premixed flame dynamics in tubes is important for a wide range of scientific and engineering scenarios, e.g., gas explosions in confined regions, combustion limits in flammability tubes, and burning processes in combustion engines. A premixed flame is intrinsically unstable due to physical phenomena such as fluid-dynamic or combustion instabilities, vortical flow, flame acceleration or deceleration, and pressure waves. Among these phenomena, pressure waves have a direct effect on flame instabilities, as shown in experiments $[1,2]$ and numerical simulations [3-5].

A flame propagating in a tube can assume a number of different shapes, e.g., spherical, curved, tulip and cellular flames. A tulip flame (TF) is a premixed flame that, as it propagates down a tube, develops a tulip-like shape with a cusp pointing towards the burned gas (see Fig. 1 at $3.8 \mathrm{~ms}$ ). There is a long history of study on TFs since they were first photographed by Ellis [6]. Subsequent studies show that TFs are robust and can form in various combustion configurations, e.g., laminar flame propagation in closed [6-8] and half-open tubes [9, 10], burning processes in combustion chambers of wave rotor [11] and wave disc engines [12], and flames propagating in a standard flammability tube [13].

Tulip flames have given rise to many studies with the purposes of explaining their physical origin [5, 7-9, 14-16]. Empirical and analytical models $[9,10]$ have also been suggested for TF evolution. From these studies, we have learned that the formation of a TF is sensitive to many parameters and 
can be produced by seemingly contradictory mechanisms. Recent experimental [16] and numerical $[17,18]$ studies show that a TF is a fluid-dynamic phenomenon arising from interactions between flame and fluid motion, instead of an instability. A similar observation was also make in our prior work [5]. The TF considered here is an inverted flame front that forms after the deceleration of a finger-shape flame in a closed tube. (See Fig. 1 at 1.98 to $3.8 \mathrm{~ms}$.) A recent detailed review of TFs is given in [5]. To summarize, basic questions about TF are: What triggers the flame inversion? How does a TF evolve as it propagates?

The recent discovery of the distorted tulip flame (DTF) further complicates the situation [5, 1921]. A DTF, which can be generated after the formation of a TF, has additional cusps or indentations superimposed on a primary flame surface. (See, for example, Fig. 1 at $5.99 \mathrm{~ms}$ and the flames in Fig. 3 in [5].) Prior experiments [19] showed that DTFs can form in hydrogen-air mixtures with equivalenceratios of $0.84 \leqslant \phi \leqslant 4.22$. In some cases, a second DTF can form just before the first DTF disappears [21]. To summarize prior work [5], we note that the TF-DTF combustion process has five stages: (1) a hemispherical flame that expands outwards, unaffected by sidewalls, (2) a finger-shaped flame which is elongated along the axis due to confinement and accompanied by a rapid increase in flame surface area, (3) a flame whose skirt touches the sidewalls, which, as a result, reduces surface area, (4) a TF which forms after flame inversion and develops a cusp pointing towards burned gas, resulting in an increase in flame surface area, and (5) DTFs with secondary cusps superimposed on the primary surface of a TF.

Prior multidimensional fully compressible numerical simulations reproduced the major features of the experimentally observed formation and evolution of DTFs, both qualitatively and quantitatively [5]. In addition, they showed that a series of DTFs formed, one after another, before the flame reached the end of the tube. One important new result was the formation and collapse of flame cusps and the consequent generation of pressure waves, which result in flame oscillations when they interact with flame front. The amplitude of the pressure waves was high enough to cause Rayleigh-Taylor instabilities (RTIs). It was suggested [5] that pressure waves may contribute to the growth of TF, although vortex motions can be a major cause. The interaction among the flame front, pressure waves, and boundary layers appeared to be essential for the initiation of DTFs. The fact that the Lewis number was 1.0 in the computations demonstrated that thermal-diffusive instabilities were not a likely candidate to explain DTF formation.

Reference [5] mainly considered the dynamics of DTFs in a closed $4 \mathrm{~cm} \times 28 \mathrm{~cm}$ tube. Although RTIs arising from the interaction between the flame front and pressure waves were suggested as a cause of DTF, it was not proven definitively since there was no supporting analysis. The conditions required 
for initiation and development of DTFs were not known either, e.g., the influence of configurations and length scales of the system under study. Questions then arise, such as: What determines the initiation and growth of a DTF? Does the formation of a DTF depend on the configuration and size of the system considered? Can we quantify the effects of the RTI on DTFs?

In the following sections we present the results from a series of two-dimensional numerical simulations of flame propagation in closed tubes. The length and acoustic time scales were varied from case-to-case by changing the geometry of tube. The results of these simulations are then analyzed using linear stability theory to show that the conditions and timing of the occurrence of DTFs are consistent with the development of unstable RTI modes, and thus support the idea that RTI is responsible for DTFs.

\section{Numerical method and physical model}

Flame propagation in a close tube is modeled as a fully compressible reacting flow (see [5]). The twodimensional (2D) reactive Navier-Stakes equations are solved using a fifth-order MUSCL algorithm [22] with an HLLC Riemann solver [23]. The time integration is advanced using a third-order explicit Runge-Kutta algorithm. The simplified chemical-diffusive model used has a reaction rate of the form,

$$
\dot{\omega}=d Y / d t=A \rho Y \exp \left(-E_{a} / R T\right)
$$

where $Y, t, A, \rho, E_{a}, R$ and $T$ are the mass fraction of unburned reactant, time, pre-exponential factor, density, activation energy, gas constant and temperature, respectively. The dynamic viscosity, mass diffusivity, and thermal diffusivity are calculated as $\mu=\mu_{0} T^{0.7}, D=D_{0} T^{0.7}$, and $k=k_{0} T^{0.7}$, respectively, where $\mu_{0}, D_{0}$ and $k_{0}$ are the transport coefficients under conditions $T_{0}=298.0 \mathrm{~K}$ and $p_{0}=1.0 \mathrm{~atm}$. The gas is assumed to be ideal with constant specific heats.

The input parameters that describe a stoichiometric mixture of hydrogen and air are given in [5]. These parameters produce a laminar flame speed, flame thickness, and flame temperature of $S_{u 0}=3.11$ $\mathrm{m} / \mathrm{s}, \delta_{L}=0.34 \mathrm{~mm}$, and $T_{b}=2141 \mathrm{~K}$, respectively. The flame thickness was calculated as the distance between fuel mass fractions of $Y=0.1$ and 0.9 .

Adaptive mesh refinement (AMR) with the Paramesh library [24] ensures that essential flow features, e.g., flame fronts, pressure waves, and boundary layers, are resolved. Prior resolution tests [5] showed that the grid resolution with a minimum grid size $d x_{\max }=1 / 640 \mathrm{~cm}(15.625 \mu \mathrm{m}$, corresponding to 22 computational cells inside the flame under initial conditions) and maximum $d x_{\max }=1 / 40 \mathrm{~cm}$ is adequate. No-slip, adiabatic boundary conditions were used on the bottom, left, and right domain 
boundaries. Half-symmetry is assumed and the upper boundary is assumed to be a symmetry line. The flame is ignited by placing a small quarter-circular pocket of hot, burned gas at 1.0 atm and 2140 $\mathrm{K}$. The ignition region has a radius of $0.5 \mathrm{~mm}$ and is centered on the point where the left wall and symmetry line meet.

Computations were performed in which the size and aspect ratio, $\alpha$, of the closed tubes were varied. Table 1 gives a list of all the cases simulated. The first set of computations considered tubes at fixed $\alpha=7.0$, but with different length scales $1 \times 7,2 \times 14,4 \times 28$, and $8 \times 56$, where units of $\mathrm{cm}^{2}$ are inferred in this notation. A second set, $4 \times 14,4 \times 28$, and $4 \times 56$, considered fixed tube height $d=4 \mathrm{~cm}$, but varied $\alpha$.

\section{Results}

Here we first describe the dynamics of flame propagation in the tube with the largest aspect ratio, $\alpha=$ 14.0. Figure 1 shows computational schlieren images of premixed hydrogen-air flame evolution in the $4 \times 56$ case. The flame behaves as a usual tulip flame $(\mathrm{TF})$ in the early stages and develops a deep cusp (see $5.12 \mathrm{~ms}$ ). The initial contact of the flame skirt with the sidewalls generates strong expansion waves (see $1.98 \mathrm{~ms}$ ). The collapse of the cusp generates another strong expansion wave (5.26 and $5.35 \mathrm{~ms})$. A distorted tulip flame (DTF) starts to form after $5.90 \mathrm{~ms}$ and develops a double-cusped, concave flame front (6.91 and $7.34 \mathrm{~ms}$ ). By this time, the pressure waves are amplified (this will be shown below), and to eventually form much stronger pressure waves.

Figure 1 shows that a flame front propagating in a tube can develop various shapes, including a semi-circular, curved, tulip, distorted tulip, and highly corrugated flames. Since pressure waves are so prominent, the Rayleigh-Taylor instability (RTI) can be significant. Shock waves are generated by coalescing acoustic waves in the later stages. (See Fig. 1 at $7.34 \mathrm{~ms}$ at about halfway between the flame tip and the right wall.) These shocks change the dynamics of the flame evolution by producing Richtmyer-Meshkov instabilities. Discussion of the flame propagation at this later stage, which is beyond that of the somewhat simplified DTF discussed here, will be presented in a future paper.

Figure 2 shows successive outlines of flame fronts taken from the calculations of all cases listed in Table 1, except the $4 \times 56$ case that was shown in Fig. 1. Flames in tubes with fixed $\alpha=7.0$ evolve with qualitatively the same pattern. TF and DTFs appear as a cascade of cusps or wrinkles with decreasing length scales as the flame propagates farther into the tube. The flame front first becomes inverted and develops a large-scale cusp with a wave length approximately equal to the tube width. As the flame continues to propagate down the tube, smaller and smaller structures form a hierarchy of cusps. The DTF is more pronounced as the tube size increases at fixed $\alpha$. The flame in the $4 \times 14$ tube with the 
smallest $\alpha=3.5$ shows the first four stages of the TF-DTF combustion process discussed in section 1 and develops a TF, but not a DTF.

\section{Discussion}

Here we first discuss the relationships between pressure waves, flame oscillations and distorted tulip flame (DTF) formation to show the importance of pressure waves for flame instabilities. Then, to demonstrate the role of Rayleigh-Taylor instability (RTI) in DTF formation, we present a simplified analysis of the RTI driven by pressure waves such as those observed in the computations.

\subsection{Oscillating flame propagation and distorted tulip flame formation}

Figures 3 and 4 show the normalized flame propagation speed, $S_{\text {tip }}^{*}=S_{\text {tip }} / C_{s}$, of the leading tip of the flame, measured in the laboratory frame of reference, and the reduced pressure $p^{*}$, computed where the symmetry line the right wall meet, all as a function of reduced time $\tau$, respectively. Here $L$ is the length of tube and $C_{s}=371.54 \mathrm{~m} / \mathrm{s}$ is the sound speed in the unburned mixture at the initial conditions. The reduced times when the tulip flame $(\mathrm{TF})$, the first, second, and third DTFs form are denoted by $\tau_{\text {tulip }}$,

$\tau_{D T F 1}, \tau_{D T F 2}$, and $\tau_{D T F 3}$, respectively. The time scale, propagation speed $S_{\text {tip }}$, and pressure $p$ are normalized by $L / C_{s}, C_{s}$, and $p_{0}$, respectively.

The reduced time starts when the flame first touches tube sidewalls at $t_{\text {wall }}$. This time is chosen as the initial time in the analysis because it is when the primary pressure waves in the system are generated by the initial contact of the flame with the sidewalls. For fixed $d=4 \mathrm{~cm}$, the values of $t_{\text {wall }}$ are very close, i.e., 1.64, 1.69 and $1.70 \mathrm{~ms}$ for $L=14,28$ and $56 \mathrm{~cm}$, respectively. This is consistent with theories in $[9,10]$, which demonstrated that $t_{\text {wall }}$ is not related to $L$, but is determined by $d, S_{u 0}$ and the density ratio.

Figure 3 shows $S_{\text {tip }}^{*}$ and $p^{*}$ for tubes with fixed $\alpha=7.0$ and different lengths. For fixed $\alpha, S_{\text {tip }}^{*}$ and $p^{*}$ almost overlap in the early stages. The reduced times for the contact of the flame with the sidewalls and formation of TF and DTFs are also close. Differences in the oscillation phase, pressure growth, and $S_{\text {tip }}^{*}$ occur in the later stages when the flame approaches the end wall. These differences arise as the flame develops more small-scale wrinkles in the larger tubes (Fig. 2), which results in a faster overall burning rate and more pressure waves.

Figure 4 shows the values of $S_{\text {tip }}^{*}$ and $p^{*}$ in the tubes with fixed $d=4 \mathrm{~cm}$ but different $\alpha$. The trends in the frequency and phase of the oscillations are similar to those seen in Fig. 3. The frequency in later stages is much higher in the tube with $\alpha=14.0$ due to the large amplification of pressure waves and the generation of shocks. The normalized TF initiation times for different $\alpha$ are not the same, i.e., 
2.76, 1.37 and 1.0 for $4 \times 14,4 \times 28$ and $4 \times 56$ tubes, respectively. Nevertheless, the normalized initiation times of the first and second DTFs (for $4 \times 28$ and $4 \times 56$ cases) are close and coincide with the flame and pressure oscillations (Fig. 4). The later DTFs form faster in the $4 \times 56$ tube due to the effect of shock waves. These results indicate that even though pressure waves are not connected with the initiation of TFs, they are closely related to the formation of the DTFs.

\subsection{Effect of aspect ratio on flame and pressure oscillations}

Figure 4 shows that pressure oscillations and amplitudes of $S_{\text {tip }}^{*}$ and $p^{*}$ increase as $\alpha$ increases. This is consistent with experiments of premixed propane-oxygen-nitrogen flames propagating in closed tubes [1], which suggested that an increase in $\alpha$ results in an increase in flame-tip speed and, consequently, the amplitude of pressure oscillations.

An increase in $\alpha$ results in an increase in the flame acceleration and the maximum $S_{\text {tip }}^{*}$ (Fig. 4a). This leads to a more elongated finger-shaped flame at the second stage and a larger reduction in flame surface area when the flame touches the sidewalls. Therefore, the amplitude of the pressure oscillation, $\Delta p$, is higher,

$$
\Delta_{p} \propto \Delta Q=\Delta\left(A_{f} S_{u} \rho_{u} q\right) \approx\left(\Delta A_{f}\right) S_{u} \rho_{u} q,
$$

where $Q, q, A_{f}, S_{u}$ and $\rho_{u}$ are the heat release rate, heat release per unit mass, flame surface area, burning velocity and unburned gas density, respectively [25]. In addition, more flame structures form as the pressure amplitude increases with increasing $\alpha$ (Figs. 1 and 2).

The flame-speed and pressure oscillations are amplified as the flame propagates down the channel due to the development of the TF and DTFs. Coalescence of pressure waves with stronger acoustic waves also contributes to the amplification. A larger $\alpha$ results in a higher initial amplitude of the pressure oscillations (Fig. 4b), and consequently greater amplification of oscillations. This is consistent with experimental observations [1], in which an initial amplitude larger than $5.0 \mathrm{kPa}$ caused continuous growth in pressure-wave amplification. For the $4 \times 56$ tube, the pressure oscillation has a much higher initial amplitude.

\subsection{Analysis of Rayleigh-Taylor instability}

RTI arises from the generation of vorticity through baroclinic effects when a curved material interface is exposed to a pressure gradient and there is a misalignment between pressure and density gradients. This is qualitatively what we see above and what we now explore quantitatively.

The acoustic mode shown in the calculations is a half-wave mode with a single pressure peak traveling back and forth inside the tube. The half wavelength of the pressure waves is equal to the 
length of the tube. The fundamental period of the longitudinal acoustic mode can be estimated by using the sound speeds for burned and unburned gases when the flame first touches the sidewalls. These periods are 0.3 and $2.74 \mathrm{~ms}$ for the shortest tube $(1 \times 7)$ and longest tube $(4 \times 56)$, respectively. These values are close to those derived from the computed pressure-time traces in Figs. 3 and 4, which are 0.29 and $2.63 \mathrm{~ms}$, respectively. This similarity holds for all of the tubes. The oscillation period deceases as the flame continues to propagate, due to the increase of sound speed accompanied by the growth of volume of burned gas and the temperature rise with compression in both burned and unburned gases.

The characteristic growth rate, $\omega$, of the RTI is

$$
\omega=\sqrt{k G\left(\rho_{f}-\rho_{p}\right) /\left(\rho_{f}+\rho_{p}\right)},
$$

where $G$ is the flow acceleration in the longitudinal direction, $k$ is the wave number, and $\rho_{f}$ and $\rho_{p}$ are the densities of unburned and burned gases [26]. The Froude number is $F r=S_{\mathrm{u}}^{2} /(G d)$, where $S_{u}$ is the laminar burning velocity. The ratio of the growth rate to the reciprocal of a half oscillation period, $\beta=\omega /\left(2 / t_{a}\right)$, is evaluated to determine if the time scale is sufficient for RTI to develop, where $t_{a}$ is the oscillation period. In the other half of the period, the pressure gradient has a stabilizing effect.

The values of $\omega, F r$, and $\beta$ during the formation of the first DTFs are given in Table 1. Here $k \approx 2 \pi /(d / 2)$. We assumed that the first DTF is two wavelengths. The acceleration $G$ is calculated as the average acceleration of flow over the acceleration time based on the computations. The growth rate is large and the Froude number is $F r \sim 10^{-3}$ in all cases, which means the acceleration is high enough for RTI to develop. The half period of the pressure-wave modes is sufficient for the development of RTI in the cases of fixed $\alpha=7.0$. For $\alpha=14.0$, the time-scale ratio is much higher and allows RTI to distort the flame to a greater extent, as shown in Fig. 1. For $\alpha=3.5$, the time scale is insufficient for RTI to develop, so that no noticeable DTF can be observed. The pressure waves act in a similar way through RTIs to form the later DTFs.

\section{Conclusions}

Numerical simulations of premixed flame dynamics in closed tubes have been performed by solving the 2D fully compressible Navier-Stokes equations coupled with a simplified chemical-diffusive model using a fifth-order MUSCL algorithm and adaptive mesh refinement [5]. Tubes with different sizes and aspect ratios were considered here to show the effects of pressure waves on the flame propagation.

In tubes with a moderate aspect ratio, $\alpha=7.0$, the tulip flame (TF) and distorted tulip flames (DTFs) evolve as a hierarchy of cusps with decreasing length scales through Rayleigh-Taylor instabilities 
(RTIs). For larger $\alpha$, the flame accelerates faster, and stronger pressure waves are generated when the flame touches tube sidewalls. This leads to more drastic flame and pressure oscillations and greater amplification of these oscillations, and, as a result, more flame structures are created. Very strong pressure waves are generated at the largest $\alpha=14.0$ considered, and cause DTFs and highly corrugated flame shapes through RTI. By contrast, the smallest $\alpha=3.5$ does not allow RTI to develop, and thus a DTF can not be created.

For fixed aspect ratio, DTFs form at close values of a nondimensional time, $\tau$, which is normalized by a characteristic acoustic time. For fixed tube diameter, flame oscillations and DTFs formation are also closely related to an acoustic time. Thus we see that acoustics and characteristic acoustic times for the system control the evolution of DTFs.

One major conclusion of this paper is that RTIs resulting from the interactions between flames and pressure waves are responsible for the formation of DTFs. This was shown by starting with linear RTI theory, which would be applicable in the early stages of the interaction between the flame and pressure waves, and which gives us theoretical growth rates, given the pressure and flame conditions. When this is compared to timings of DTF formation in the simulations, we see that there is enough time for the RTI to arise and cause the major perturbations to the flame shape that we observe.

\section{Acknowledgment}

This work was supported in part by the University of Maryland (UMD) through Minta Martin Endowment Funds in the Department of Aerospace Engineering, and through the Glenn L. Martin Institute Chaired Professorship at the A. James Clark School of Engineering. Parts of this work were funded by the Office of Naval Research. The authors acknowledge the UMD supercomputing resources (http://www.it.umd.edu/hpcc) made available in conducting the research reported in this paper.

\section{References}

[1] J. C. Leyer, N. Manson, Proc. Combust. Inst. 13 (1971) 551-558.

[2] G. Searby, Combust. Sci. Technol. 81 (1992) 221-231.

[3] O. J. Teerling, A. C. McIntosh, J. Brindley, V. H. Y. Tam, Proc. Combust. Inst. 30 (2005) 17331740.

[4] A. Petchenko, V. Bychkov, V. Akkerman, L.-E. Eriksson, Phys. Rev. Lett. 97 (2006) 164501.

[5] H. Xiao, R. W. Houim, E. S. Oran, Combust. Flame 162 (2015) 4084-4101. 
[6] O. C. d. C. Ellis, J. Fuel Sci. 7 (1928) 502-508.

[7] D. Dunn-Rankin, P. K. Barr, R. F. Sawyer, Proc. Combust. Inst. 21 (1986) 1291-1301.

[8] M. Matalon, P. Metzener, J. Fluid Mech. 336 (1997) 331-350.

[9] C. Clanet, G. Searby, Combust. Flame 105 (1996) 225-238.

[10] V. Bychkov, V. Akkerman, G. Fru, A. Petchenko, L. E. Eriksson, Combust. Flame 150 (2007) 263-276.

[11] D. Baronia, M. R. Nalim, P. Akbari, AIAA Paper (2007) 5054.

[12] Y. M. Najim, N. Mueller, I. S. Wichman, Combust. Flame 162 (2015) 3980-3990.

[13] J. Jarosinski, R. Strehlow, A. Azarbarzin, Proc. Combust. Inst. 19 (1982) 1549-1557.

[14] F. S. Marra, G. Continillo, Proc. Combust. Inst. 26 (1996) 907-913.

[15] J. W. Dold, G. Joulin, Combust. Flame 100 (1995) 450-456.

[16] B. Ponizy, A. Claverie, B. Veyssire, Combust. Flame 161 (2014) 3051-3062.

[17] A. Hariharan, I. S. Wichman, Combust. Sci. Technol. 186 (2014) 1025-1040.

[18] A. Hariharan, I. S. Wichman, Combust. Sci. Technol. 187 (2015) 1562-1583.

[19] H. Xiao, Q. Wang, X. He, J. Sun, X. Shen, Int. J. Hydrogen Energy 36 (2011) 6325-6336.

[20] H. Xiao, D. Makarov, J. Sun, V. Molkov, Combust. Flame 159 (2012) 1523-1538.

[21] H. Xiao, Q. Wang, X. Shen, S. Guo, J. Sun, Combust. Flame 160 (2013) 1725-1728.

[22] B. Thornber, A. Mosedale, D. Drikakis, D. Youngs, R. J. R. Williams, J. Comput. Phys. 227 (2008) $4873-4894$.

[23] E. F. Toro, M. Spruce, W. Speares, Shock Waves 4 (1994) 25-34.

[24] P. MacNeice, K. M. Olson, C. Mobarry, R. d. Fainchtein, C. Packer, Comput. Phys. Commun. 126 (2000) 330-354.

[25] B.-T. Chu, Proc. Combust. Inst. 4 (1953) 603-612.

[26] D. H. Sharp, Physica D 12 (1984) 3-18. 


\section{Figure captions}

Figure 1. Sequence of numerical schlierens showing the evolution of premixed stoichiometric hydrogenair flame and strong pressure waves interacting with the flames in the $4 \times 56 \mathrm{~cm}^{2}$ closed tube. Each time instance is mirrored about the symmetry line at $y=0$.

Figure 2. Evolution of the surface of flames propagating in closed tubes with different sizes and aspect ratios, $\alpha$. Successive frames are taken every 11000, 21000, 38000, 60000, and 34000 time steps, for the tubes of $1 \times 7 \mathrm{~cm}^{2}, 2 \times 14 \mathrm{~cm}^{2}, 4 \times 28 \mathrm{~cm}^{2}, 8 \times 56 \mathrm{~cm}^{2}$, and $4 \times 14 \mathrm{~cm}^{2}$, respectively. The flame surfaces are mirrored about the symmetry line.

Figure 3. Normalized propagation speed of flame leading tip $S_{\text {tip }}^{*}(\mathrm{a})$, and normalized pressure rise (b) as a function of normalized time in closed tubes with fixed aspect ratio $\alpha=7.0$. The reduced time starts when the flame touches the tube sidewalls.

Figure 4. Normalized propagation speed of flame leading tip $S_{\text {tip }}^{*}$ (a), and reduced pressure rise (b) as a function of reduced time in closed tubes of fixed width $d=4 \mathrm{~cm}$ at different aspect ratios. The notations $\tau_{D T F 1}$ and $\tau_{D T F 2}$ are for the tubes $4 \times 28 \mathrm{~cm}^{2}$ and $4 \times 56 \mathrm{~cm}^{2}$. 
Table 1. Characteristics of tubes simulated

\begin{tabular}{llllllll}
\hline & & \multicolumn{5}{c}{ Tube size $(d \mathrm{~cm} \times L \mathrm{~cm})$} \\
\cline { 3 - 7 } Symbol & Description & $1 \times 7$ & $2 \times 14$ & $4 \times 28$ & $8 \times 56$ & $4 \times 14$ & $4 \times 56$ \\
\hline$\alpha$ & Aspect ratio & 7.0 & 7.0 & 7.0 & 7.0 & 3.5 & 14.0 \\
$t_{a}$ & Acoustic period $(\mathrm{ms})$ & 0.25 & 0.54 & 1.09 & 2.11 & 0.42 & 1.72 \\
$G$ & Acceleration $\left(\times 10^{3} \mathrm{~m} / \mathrm{s}^{2}\right)$ & 424 & 200 & 99 & 49 & 80 & 199 \\
$\omega$ & RTI growth rate $\left(\times 10^{3} \mathrm{~s}^{-1}\right)$ & 20.1 & 9.7 & 4.8 & 2.4 & 4.4 & 6.9 \\
$F r$ & Froude number $\left(\times 10^{-3}\right)$ & 2.28 & 2.42 & 2.44 & 2.49 & 3.03 & 1.21 \\
$\beta$ & Time-scale ratio & 2.51 & 2.63 & 2.64 & 2.53 & 0.91 & 5.92 \\
\hline
\end{tabular}


Figure 1

time (ms)

0.44 )

1.63

1.98

3.21

3.80

4.34

5.12

5.26

5.35

5.73

5.99

6.30

6.53

6.91

7.34

7.57
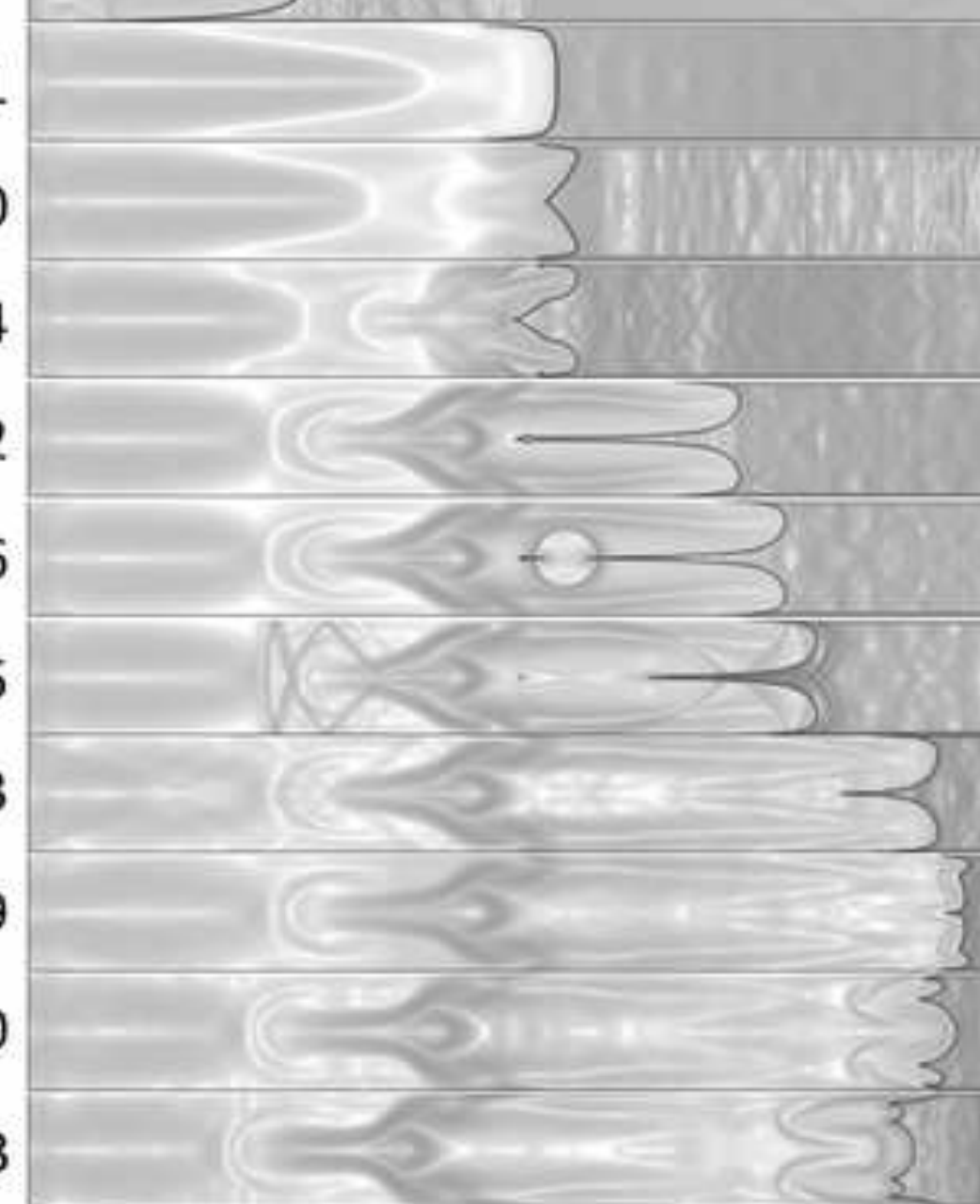

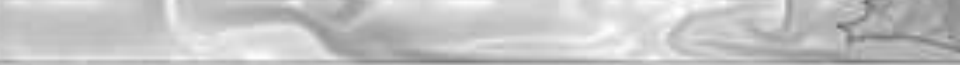


$1 \times 7 \mathrm{~cm}^{2}$

$\alpha=7.0$

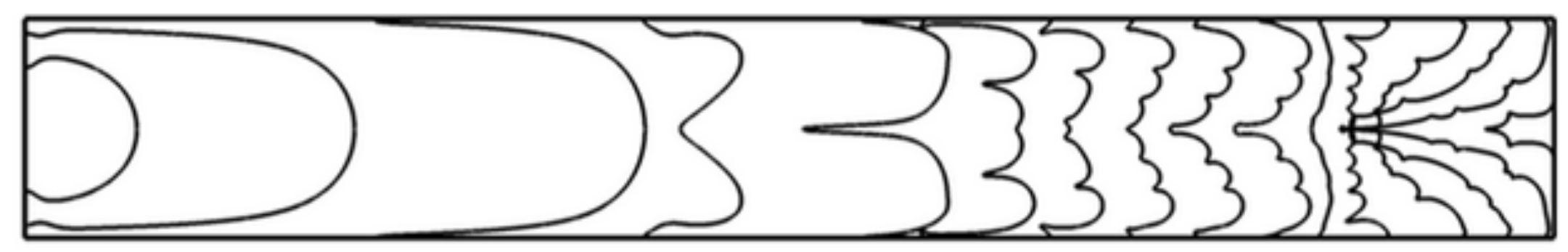

$2 \times 14 \mathrm{~cm}^{2}$

$\alpha=7.0$

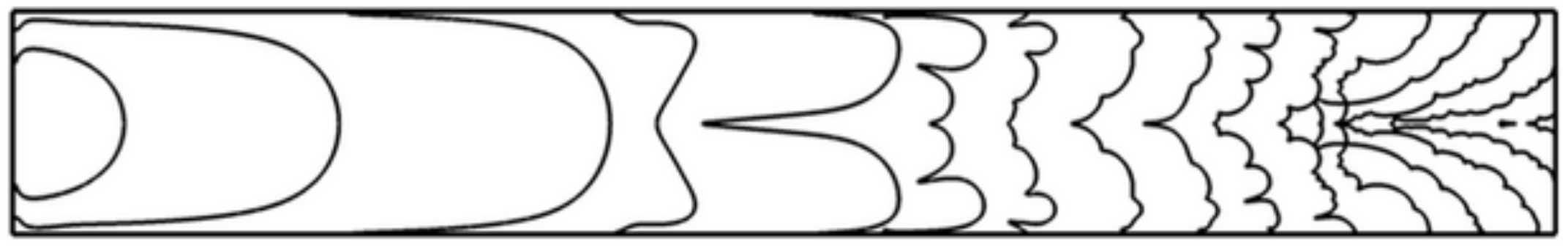

$4 \times 28 \mathrm{~cm}^{2}$

$\alpha=7.0$

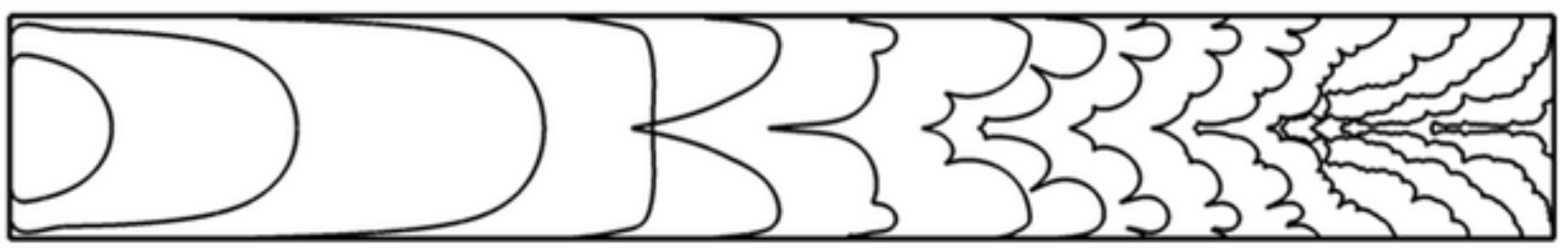

$8 \times 56 \mathrm{~cm}^{2}$

$\alpha=7.0$

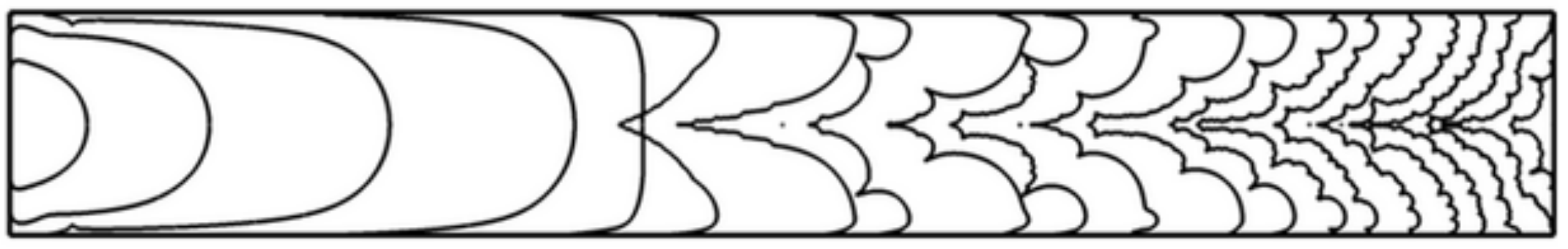

$4 \times 14 \mathrm{~cm}^{2}$

$\alpha=3.5$

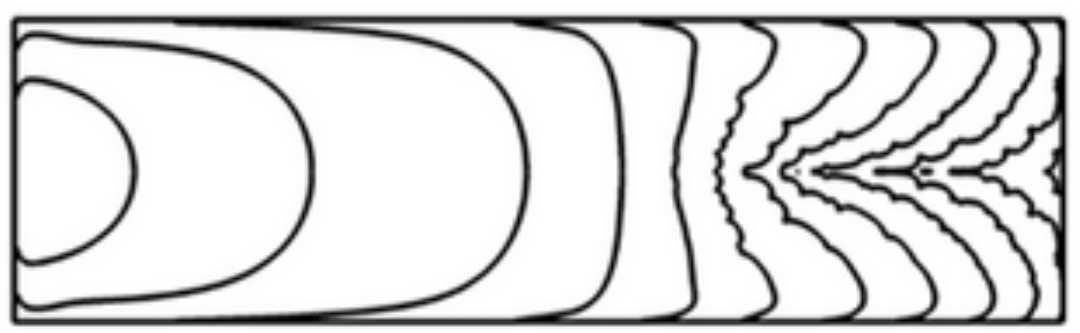



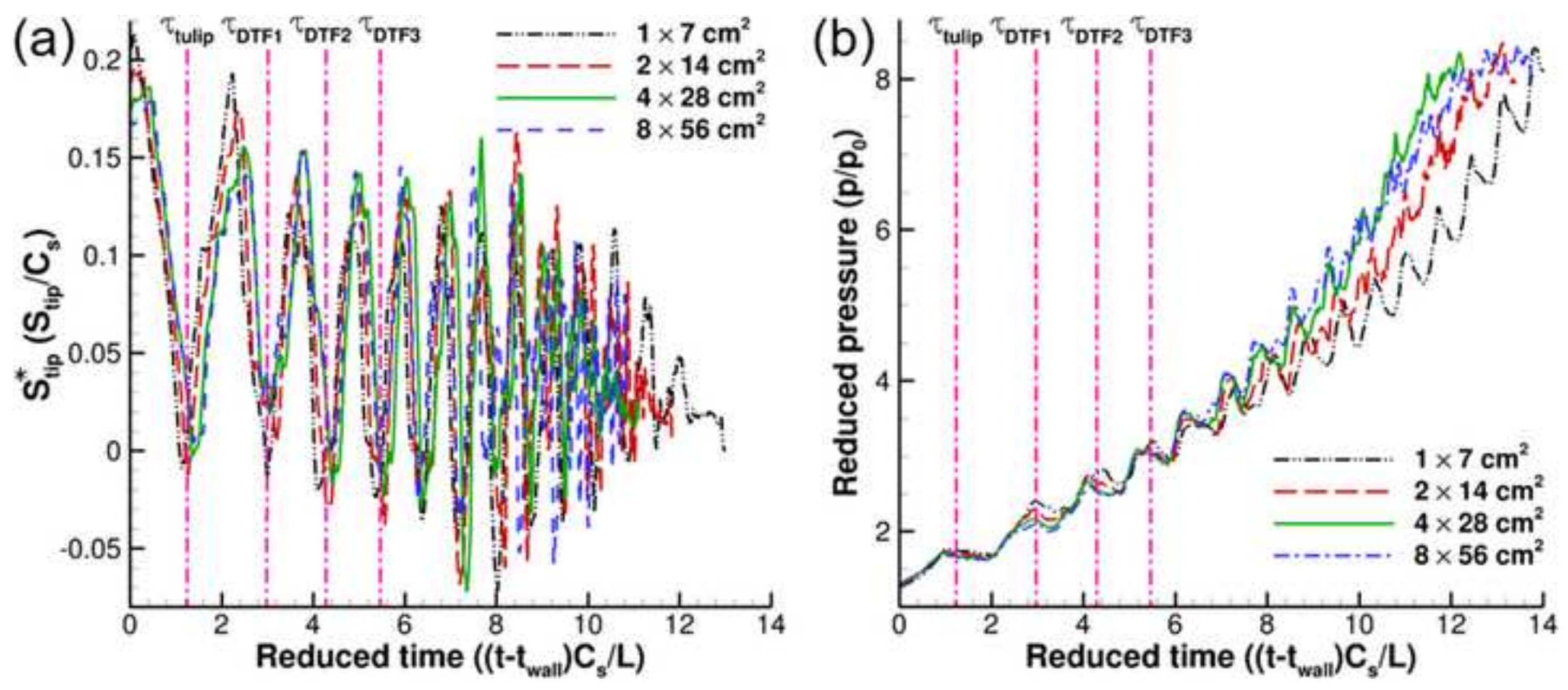

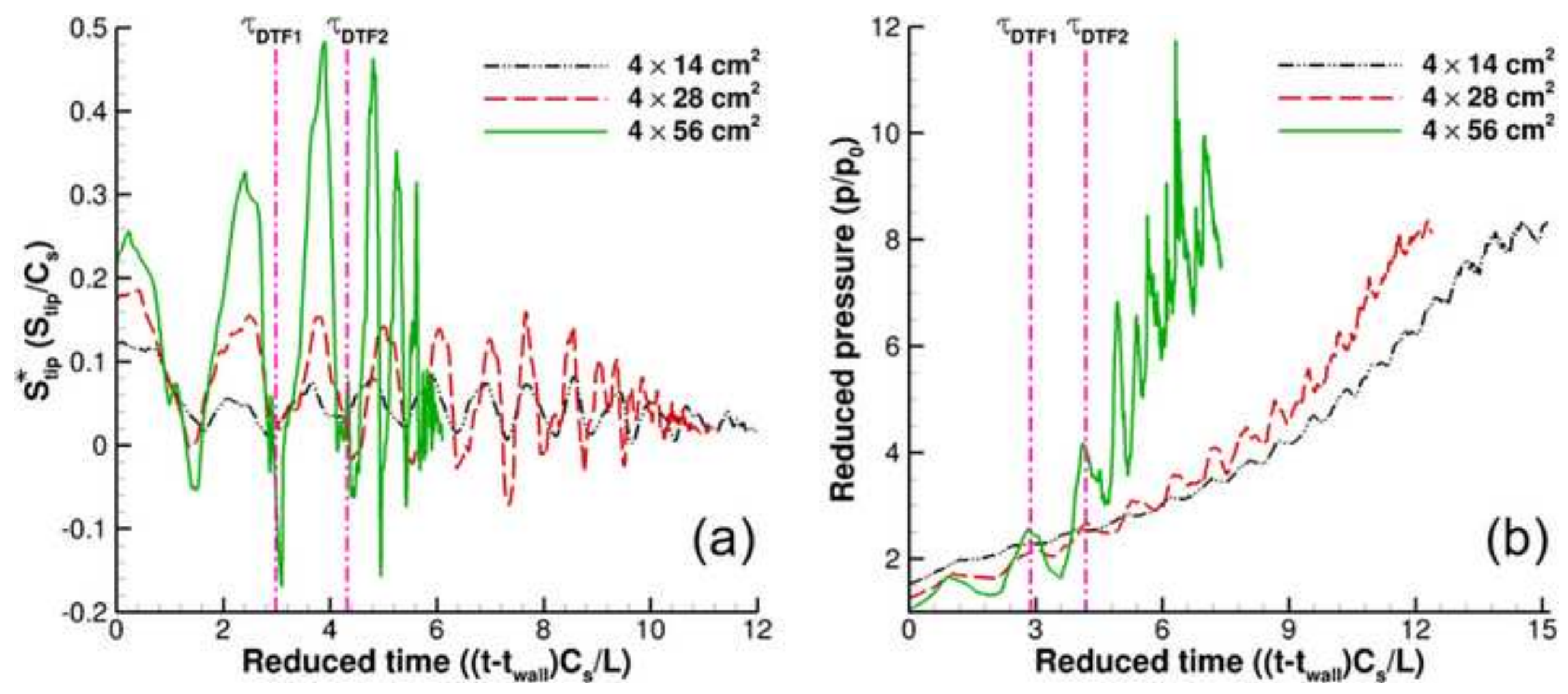

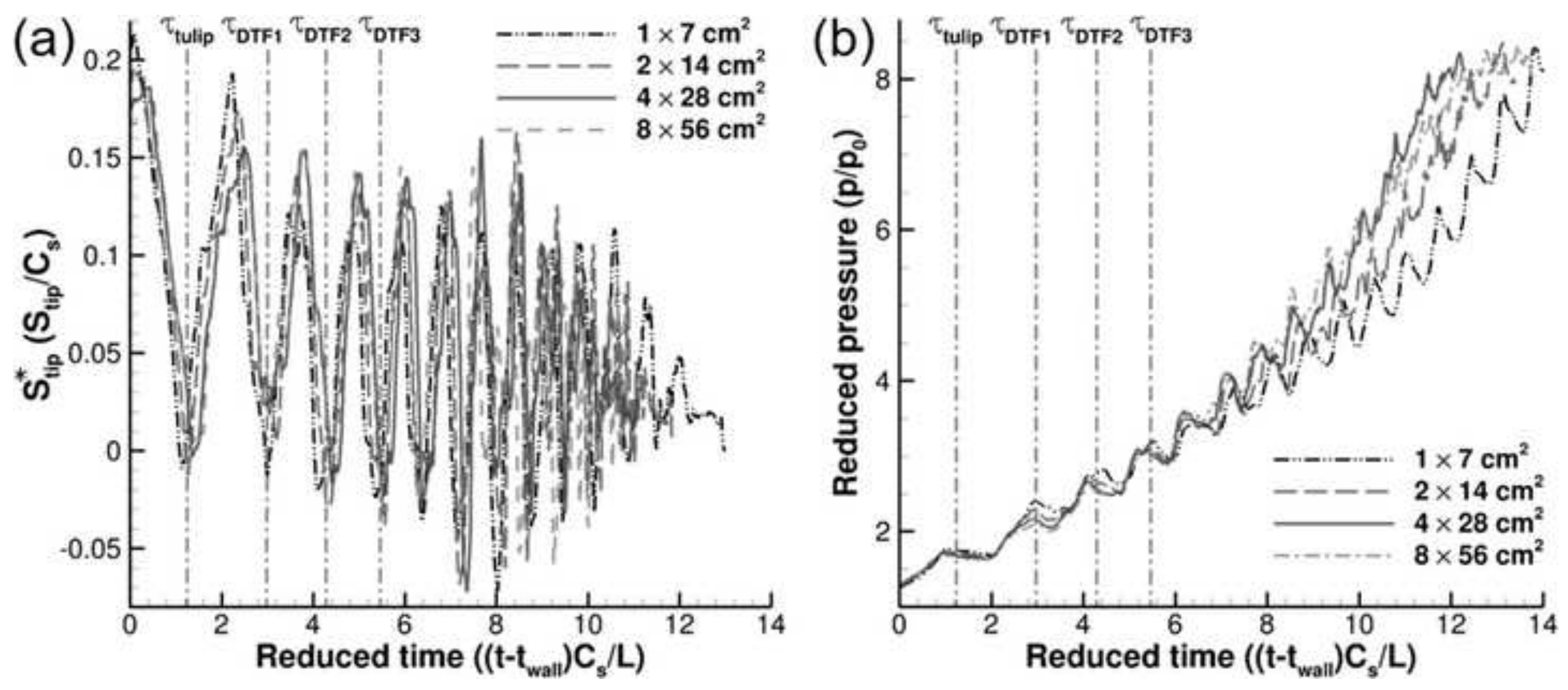

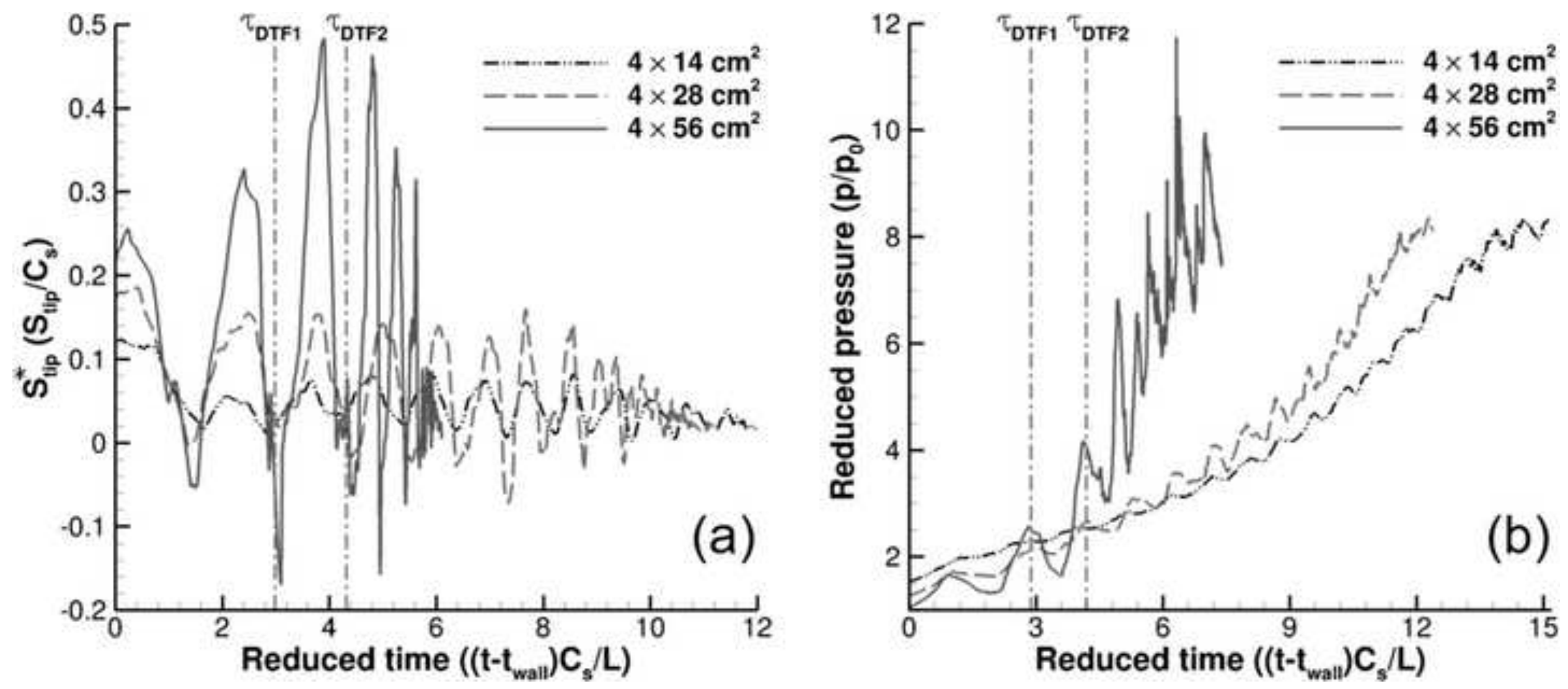